\title{
Design and Construction of an Obstacle-Detecting Glasses for the Visually Impaired
}

\author{
Adegoke A. O ${ }^{a}$, Oluseun D Oyeleke ${ }^{b}$, Mahmud B ${ }^{a}$, Ajoje J. O ${ }^{a}$, Sadiq Thomase ${ }^{b}$ \\ a, Eastern Mediterranean University, ,Famagusta, 99628, North Cyprus \\ ${ }^{b}$ Nile University of Nigeria, Abuja FCT, 900001, Nigeria
}

Received: 04 April 2019; Accepted: 12 June 2019; Published: 08 July 2019

\begin{abstract}
This paper introduces a wearable eyeglass with an ultrasonic sensor to help the blind to navigate alone safely, while avoiding obstacles that may be encountered, fixed or mobile, thereby preventing any possible accident. The main component of this work is the ultrasonic sensor, which is used to measure the distance to an object using sound waves. By recording the elapsed time between the wave being generated and the wave bouncing back, it is possible to calculate the distance between the sensor and the object. A limitation of the project is that even though it can operate alone as an independent device, its full functionality to be achieved, it needs to be part of a whole system hence making it an auxiliary device. An integration of this device with a smart walking stick or an automatic wheelchair can help realize its full potential and make it the ultimate mobility aid.

This system is cheap, fast and easy to use and it is an innovation for the blind and visually impaired people to help them overcome the various problems in their daily life.
\end{abstract}

Index Terms: Visually impaired people, Ultrasonic sensor, Wearable eyeglasses, Obstacle detection, Mobility aid.

(C) 2019 Published by MECS Publisher. Selection and/or peer review under responsibility of the Research Association of Mode rn Education and Computer Science

* Corresponding author.

E-mail address: 


\section{Introduction}

Sensors are devices whose function is solely the detection of some kind of input signals from the physical surroundings or environment, and respond appropriately to the input signal and situation. These input signals could be heat, light, motion, pressure or any other from a number of environmental phenomena. The response of a sensor, the output, is usually a signal further processed through its transmission electronically over a network, or it is converted into a human-readable display signal at the sensor location [1]. A sensor typically sends out certain kinds of energy including ultrasonic waves or light beams, and detects if an object, in the path of transmission, is interrupting the energy flow [2].

In light of the exponential growth in population and industrialization in the urban areas, the physically disabled have to rely more on the able individuals for their own daily activities, thereby becoming more of a liability than just another individual. This increase in dependence on a certain group of people and continuous tending to needs by another could be extremely difficult to bear for those individuals being relied on. This is what the obstacle detecting eyeglasses have come to counter. They will enable the visually impaired to move independently and actually find their way on certain little things than having to burden those without disability for these minor needs they might want to fulfill.

\subsection{Contributions of this Paper}

This work will show how sensors will revolutionize the way people see the visually impaired and open a new gateway to more productive impacts on the society from them, and also how it can spark a series of inventions and discoveries in order to help other disabled communities too become more useful to the society and less of a deadweight.

Compared to previous works in this area, this project allows for flexibility and integration with other devices that serve a similar purpose of being mobility aids. It has no complex functions or interface that might cause distress for the user, as it is very easy to use and operate. This device comprises of lightweight materials that do not cause stress or weariness on the wearer. It is very cheap and easy to produce and even more marketable as compared to its counterparts in the market.

\section{Literature Review}

A sensor is a device that detects in changes in quantities. The specific quantity could be light, heat, motion, distance, gases and pressure. It provides a corresponding output, generally as an electrical something that can be controlled. Most of the sensors in use today are able to communicate with an electronic device, which are going to do the measuring, calculation and recoding.

The first invented sensor was electric thermostat type sensor by Warren Johnson, which came to the market in 1883 [3]. This thermostat was able to keep temperatures within a degree of accuracy. Later, in 1940 the infrared sensors were introduced. Motion sensor invention followed this, which was used for an alarm system, by Samuel Bango. This sensor made use of ultrasonic frequencies to determine the location of a person in a room and then it was used to calculate the movement of the stars. These motion sensors widely used in World War II with mine. Mines placed below the ground would explode when it sense movement on the surface. In modern technology it is used at Radar to track enemies and helps in air traffic control to avoid airplane accidents [4].

In the visually disabled persons research area, there have been different developments made on smart-glasses and ultrasonic sensors. Mohammed H. Mahmud, Rana Saha and Sayemul Islam came up with a Smart walking stick to assist visually impaired individuals with a basic mobility aid using a microcontroller [5]. The hardware consists of a microcontroller incorporated with ping sonar sensor, distance sensor, wet detector, a 
micro pager motor and additional equipment. This blind aid system can be designed to take of pattern of a portable and classic device, which can be mounted on an ordinary white cane or blind stick. Another research by Jinqiang Bai, Shiguo Lian, Zhaoxiang Liu, Kai Wang and Dijun Liu brought about a simple Multi-Sensor Fusion-Based Obstacle-Detecting algorithm to be proposed [6]. It utilizes both the depth sensor and ultrasonic sensor to solve the problems of detecting small obstacles, and transparent obstacles as well. Further research by Dania Abdul-Rasool and Susan Sabra brought about Mobile-Embedded Smart Guide for the Blind [7]. This Smart Guide integrates several various technologies packed in a portable compartment consisting of a Bluetooth antenna, a sensor, a central processing unit, a memory and speakers. The device sensor sends a signal to the mobile application when it detects any solid objects in the path of movement. The mobile device then notifies the wearer and gives a warning alarm to them to change their direction and get on a proper path. Interviews and surveys with doctors and a group of blind individuals in an organization for the disabled in Kuwait, where it is currently patented, helped obtaining a very positive feedback on the success of using the device. Rohit Agarwal, Nikhil Ladha, Mohit Agarwal et al. came up with a device that includes a pair of glasses and an obstacle detection module fitted in it in the center. Coupled along is a processing unit, a beeping device, and a power supply. The Obstacle detection module and the output device is connected to the processing unit. With the main aim of this glasses being easy to use, light weight, user friendly and at cheap in price, the glasses plainly help them avoid obstacles [8]. Feng Lan, Guangtao Zhai, and Wei Lin wrote a paper about a smart glasses system with voice input for instructions and hints through wireless bone conduction headphones. They claimed that the system will help visually impaired people gain increased independence in their everyday lives [9].

\section{Design and Analysis}

In the proposed system we introduce an ultrasonic sensor and its importance for design of spectacles models for helping the blind persons. Ultrasonic sensor is used to detect the obstacles in the path of the blind, we use Power supply at sensor section, which is used for supplying electrical or other types of energy to an output load, or group of loads is called a power supply unit or PSU [10].

The present system uses an on-board minicomputer named as AVR 8-bit microcontroller, which consists of number of input and output ports. The input and output port of the micro controller are interfaced with different input and output modules depending on the requirements [11]. The current system uses Ultrasonic sensors module, as input to the microcontroller and the buzzer as output module. In other words, the microcontroller acts as a communication medium for all the modules involved in the project. The system also performs a health check indication of the microcontroller using LED indicators [12].

\subsection{Analysis}

This section presents how the device works and which patterns have been developed to inform the user of its own localization. Obstacle detection is one of the main problems to solve to ensure safe navigation for blind users. We use the multi sensor architecture of our system to develop new obstacle avoidance abilities.

The process starts when power is supplied to the device. As the device boots up its operating system, it triggers the ultrasonic sensor to start sending burst signal. All the sensors are triggered at approximately the same time thus, there is very less delay. After the signal returns back to the receiver of the sensor as an echo, the device calculates the time taken from transmitting and receiving the echo. Using this time we calculate the distance of an obstacle from any of the sensors. Next, it checks if any of the distance calculated is less than the minimum distance specified that is $0.7 \mathrm{~m}$ in our case. If none of the sensors have distance less than the minimum distance, the entire process starts again. However, even if one of the sensors detects distance less than $0.7 \mathrm{~m}$, it triggers the pre-defined conditions. 


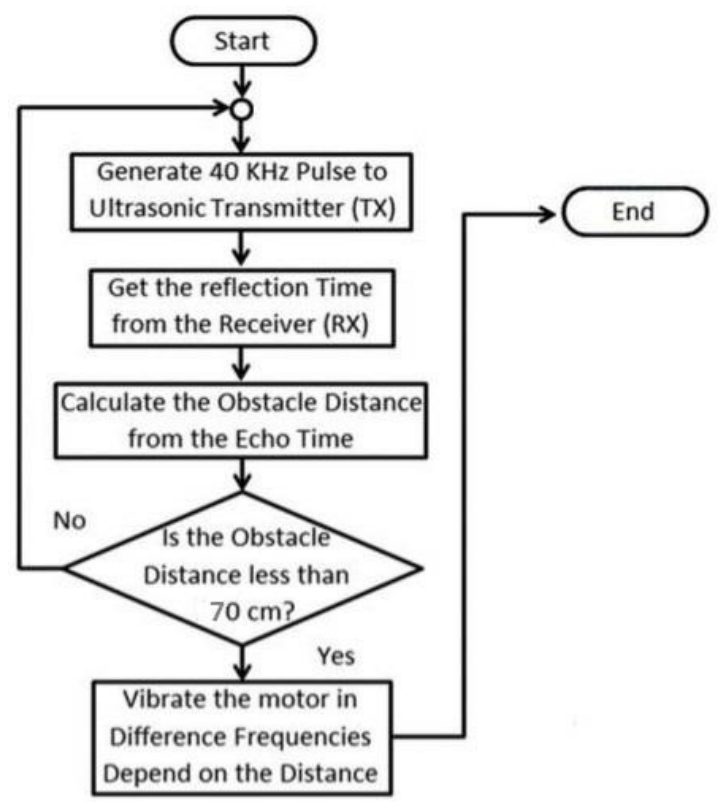

Fig.1. Flowchart of the Working Principle

The ultrasonic sensor we use here is HC-SR04 which contains 4-pins. To first pin power supply is given, the second pin is connected to P2.1 pin of controller to trigger ultrasonic rays, fourth pin is connected to ground and third is connected to P2.2 pin of controller which receives echo's when any obstacle is detected by ultrasonic rays the rays gets reflected and reflected rays is received by the this third pin and gives indication to controller obstacle is present in that direction. Once it detects the obstacle the controller will give voice alert information through voice and play back circuit. Thus we can easily identify any obstacle present around the blind people.

\subsection{Processing and Control System}

Ultrasonic sensors work on a principle similar to sonar, which evaluates attributes of a target by interpreting the echoes from sound waves respectively. Ultrasonic sensors generate high frequency sound waves and evaluate the echo, which is received back by the sensor. The time interval between the sent signal and received signal is determined to measure the distance from an object [13].

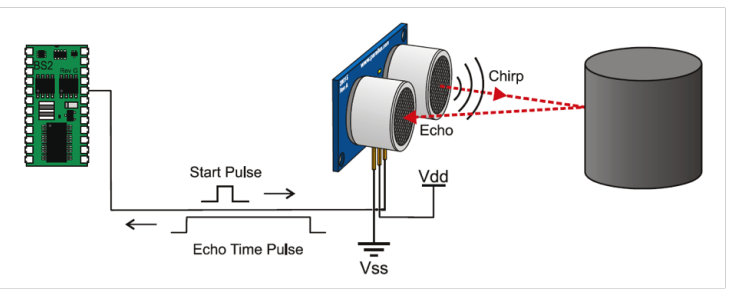

Fig.2. Working Principle of the Ultrasonic Sensor 
The HC-SR04 Ultrasonic Module has 4 pins, Ground, VCC, Trig and Echo. The Ground and the VCC pins of the module needs to be connected to the Ground and the 5 volts pins on the Arduino Board respectively and the trig and echo pins to any Digital I/O pin on the Arduino Board.

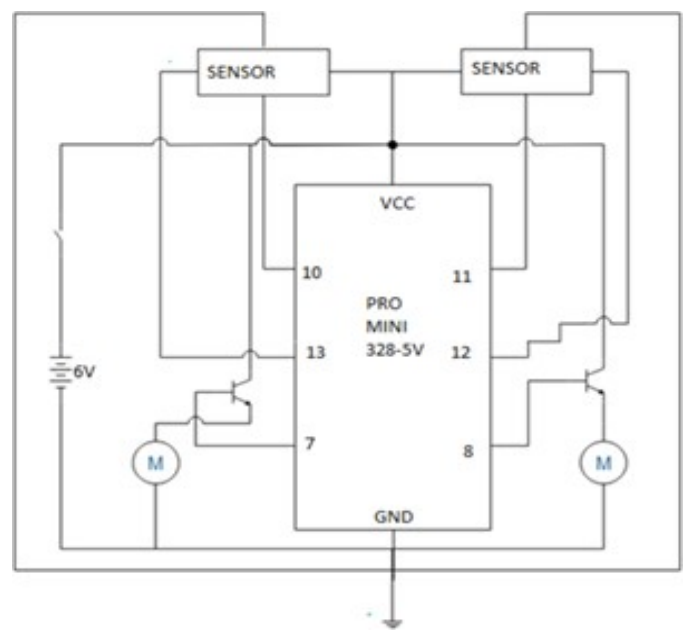

Fig.3. Schematic Diagram of Design

\section{Results Analysis}

This project looks at an obstacle detector that is easy-to-use, with minimal effort, and lightweight for visually impaired persons.

In the figure given below, we can see that with the assistance of two ultrasonic range sensors (5) on each side of the glasses giving haptic feedback on the relative distances on both sensors through the two vibrating motors (4\&6), in order to assist and allow the wearer to position him/herself towards the center of an indoor passage. These connections are possible through the presence of the Arduino Pro Mini Chip (1), with power source from the battery (2). The switch (3) is added to the system in order to turn off the power of the system, when the gadget is not in use. Finally, transistors $(7 \& 8)$ are used to make the motor produce vibration.

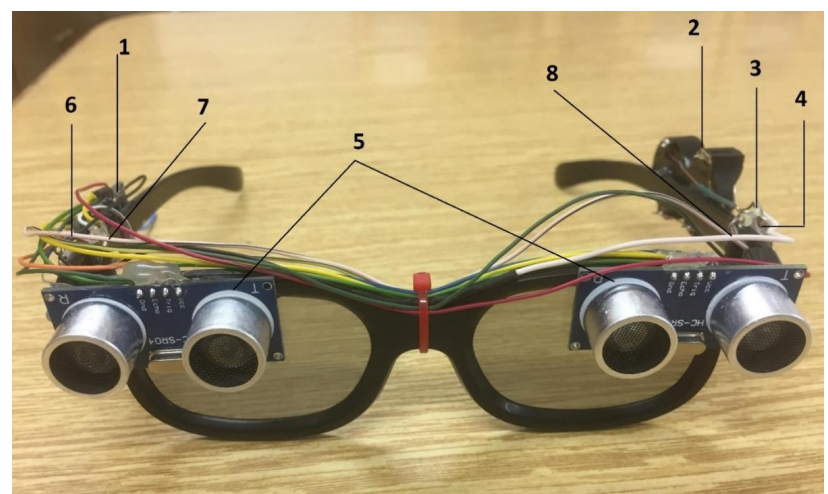

Fig.4. Frame Design and Positioning of component 


\subsection{Test results}

The tests run on this device were successful as they achieved the following results:

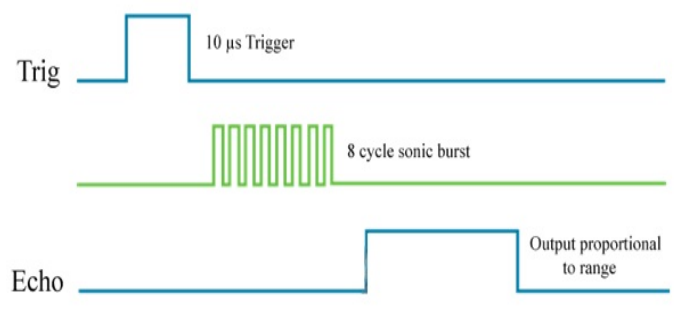

Fig.5. Trigger and Echo Relation 1

As observed from figure 3, in order to generate the ultrasound you need to set the Trig on a High State for $10 \mu \mathrm{s}$. That will send out an 8 cycle sonic burst that will travel at the speed sound and it will be received in the Echo pin. The Echo pin will output the time in microseconds the sound wave travelled.

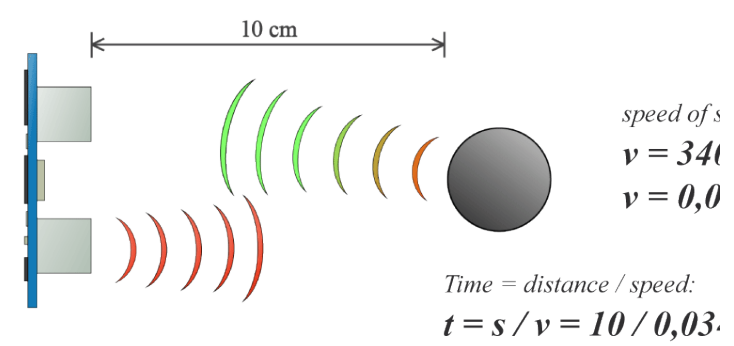

Fig.6. An Example of Trigger and Echo Relationship

For example, we noticed from figure 4 that if the object is $10 \mathrm{~cm}$ away from the sensor, and the speed of the sound is $340 \mathrm{~m} / \mathrm{s}$ or $0.034 \mathrm{~cm} / \mu \mathrm{s}$ the sound wave will need to travel about $294 \mathrm{u}$ seconds. But what you will get from the Echo pin will be double that number because the sound wave needs to travel forward and bounce backward. So in order to get the distance in $\mathrm{cm}$, we need to multiply the received travel time value from the echo pin by 0.034 and divide it by 2 .

Table 1. A table showing the variation of Intensity and frequency with respect to change in distance

\begin{tabular}{|c|c|c|}
\hline Distance & Vibration Intensity & Freq. \\
\hline$<2 \mathrm{~cm}$ & No vibration & -- \\
\hline $30 \mathrm{~cm}$ & High & $208.33 \mathrm{~Hz}$ \\
\hline $50 \mathrm{~cm}$ & Medium & $133.33 \mathrm{~Hz}$ \\
\hline $70 \mathrm{~cm}$ & Low & $83.33 \mathrm{~Hz}$ \\
\hline$>70 \mathrm{~cm}$ & No vibration & -- \\
\hline
\end{tabular}


From Table 1, it can be seen that the closer the object to the sensor, the higher the intensity and frequency of vibration of the motor. From $2 \mathrm{~cm}$ to $35 \mathrm{~cm}$, high intensity was recorded, and $65 \mathrm{~cm}$ to $70 \mathrm{~cm}$ gave low vibration intensity. Distances less than $2 \mathrm{~cm}$ and greater than $70 \mathrm{~cm}$ are out of range and therefore, no vibration will occur.

Table 2. A table showing the variation of speed and corresponding response with respect to change in surface

\begin{tabular}{|c|c|c|}
\hline Surface & Speed in Media & Response \\
\hline Glass/Hard & $4540 \mathrm{~m} / \mathrm{s}$ & Very Fast \\
\hline Wood & $3960 \mathrm{~m} / \mathrm{s}$ & Fast \\
\hline Person/Tissue & $1540 \mathrm{~m} / \mathrm{s}$ & Medium \\
\hline
\end{tabular}

From Table 2, we notice that the harder the surface/object, the faster the time of response of the sensor. Some surfaces (soft/permeable) can absorb some or part of the transmitted wave and make the system response slow, while some surfaces (solid/hard) reflect the transmitted wave instantaneously.

\subsection{Review of Test Results}

1. All the components of the device worked in cohesion and no errors recorded by the sensor from the computer readings.

2. The device was able to measure the distance with every turn at ultrasonic speed and in turn, sent a feedback to the Arduino, which triggered the motors in due process.

3. As programmed on the microcontroller, whenever an object was with $2-70 \mathrm{~cm}$ from the user, the motors vibrated. As the obstacles get nearer, the frequency of vibration increases.

4. Stair location was conceivable with the gadget. A proximity alarm cautioned the user, to keep them from running into obstacles.

5. The power supply battery lasts for 2 hours plus standby time. This is the area where improvements need to be made.

The given figure below displays the side view of the arrangement of some of the components of the obstacle-detecting glasses.

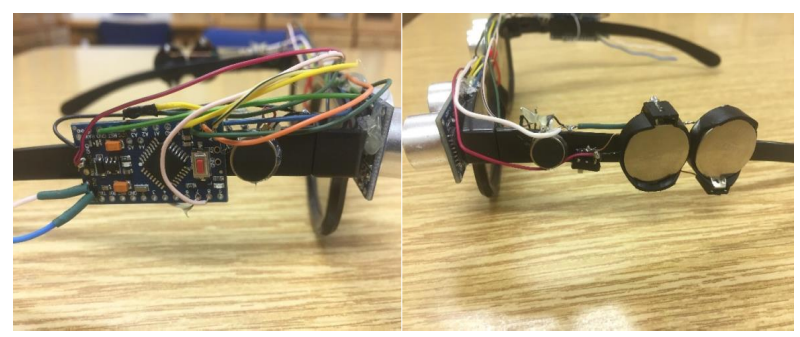

Fig.7. Side view of the Glasses

\section{Conclusion}

In this paper, the designed system uses the integration of sensors and motors to help the visually impaired navigate through obstacle while traversing. This system is fashioned to perform the action of 
obstacle detection and also warn of the proximity to detected obstacle. It serves as what is called a 'Mobility Aid', to assist those who are visually disabled, have what is close to a normal lifestyle and perform certain daily activities independently. With this in mind, the information system is simple, portable and easy to use. With some improvements in the hopes to extend the up time and make it last longer. There is also works in progress to add voice feedback to the users so as to still make things easy for them. These upgrades would include a GPS tracker and Bluetooth function so as to be integrated into an automated wheelchair and the technology can only skyrocket from there.

To conclude, it is clear that not all users will be able to grasp this kind of technology, as most of the mobility still relies mainly on their feeling of their environment and through natural instincts. However, the project and research can actually open up the flood gates to new sets of advancement in human development as it aims to bring disabled people back into the society, as most they have become a discriminated section of the modern day community.

\section{References}

[1] Rouse, M. (2010). What is sensor? - Definition from WhatIs.com. [online] WhatIs.com. Available at: https://whatis.techtarget.com/definition/sensor [Accessed 10 Jan. 2018].

[2] Thonti, V. (2018). Different Types of Sensors and their Working. [online] Circuitdigest.com. Available at: https://circuitdigest.com/tutorial/different-types-of-sensors-and-their-working [Accessed 10 Jan. 2018].

[3] What-is-a-sensor.com. (n.d.). History of Sensors. [online] Available at: http://what-is-a-sensor.com/history-of-sensors/ [Accessed 20 Nov. 2017].

[4] Ali, A. (n.d.). History of sensors. [online] Slideshare.net. Available at: http://www.slideshare.net/AbdallaAli7/history-of-sensors [Accessed 16 Nov. 2017].

[5] Mahmud, M. H., Saha, R., \& Islam, S. (2013). Smart walking stick-an electronic approach to assist visually disabled persons. International Journal of Scientific \& Engineering Research, 4(10), 111-114.

[6] J. Bai, S. Lian, Z. Liu, K. Wang and D. Liu, "Smart guiding glasses for visually impaired people in indoor environment," in IEEE Transactions on Consumer Electronics, vol. 63, no. 3, pp. 258-266, August 2017.

[7] AbdulRasool D., Sabra S. (2011) Mobile-Embedded Smart Guide for the Blind. In: Cherifi H., Zain J.M., El-Qawasmeh E. (eds) Digital Information and Communication Technology and Its Applications. DICTAP 2011. Communications in Computer and Information Science, vol 167. Springer, Berlin, Heidelberg.

[8] Agarwal, R., Ladha, N., Agarwal, M., Majee, K. K., Das, A., Kumar, S., ... \& Dey, R. (2017, October). Low cost ultrasonic smart glasses for blind. In 2017 8th IEEE Annual Information Technology, Electronics and Mobile Communication Conference (IEMCON) (pp. 210-213). IEEE.

[9] Lan, F., Zhai, G., \& Lin, W. (2015, November). Lightweight smart glass system with audio aid for visually impaired people. In TENCON 2015-2015 IEEE Region 10 Conference (pp. 1-4). IEEE.

[10] Xiangjun Z., Shaodong Y. and Le Ling, "Multimedia sensor networks design for smart home surveillance," Control and Decision Conference, 2008, Chinese, 2-4 July 2008, pp. 431-435.

[11] Benjamin J. M., Ali N. A., A laser cane for the blind In Proceedings of the San Diego Biomedical Symposium, volume 12, pages 53-57, 1973.

[12] Pascolini D, Mariotti SPM. Global estimates of visual impairment: 2010. British Journal Ophthalmology Online First published December 1, 2011 as 10.1136/bjophthalmol-2011- 300539

[13] Arduino Sensors. Retrieved from Arduinosensors.com. (n.d.). Arduino Sensors. [online] Available at: http://arduinosensors.com/index.php/arduino- ultrasonic-distance-sensor/ [Accessed 16 Nov. 2017]. 

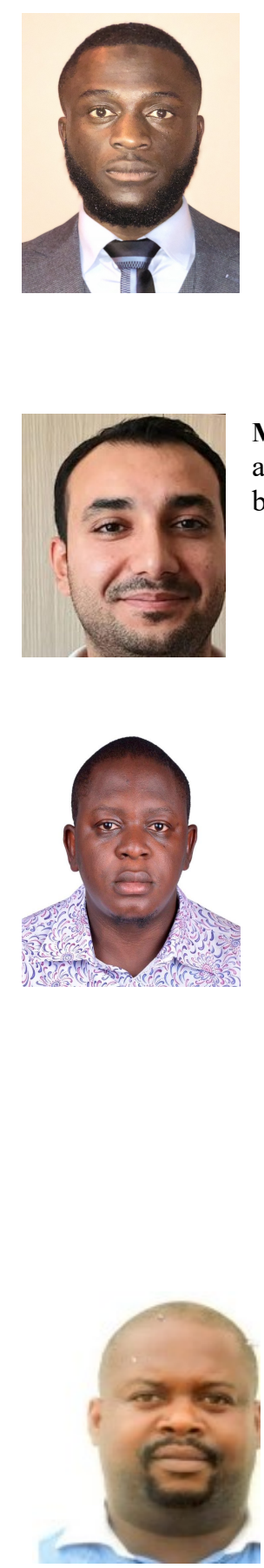

Abdullahi O. Adegoke: Currently a Master's student at Nile University. He had his BSc. from Eastern Mediterranean University in 2018.

Mahmud Bulacov holder a degree in Electrical and Electronics Engineering currently works as an Instrumentation and Control Systems Engineer in Tekfen Engineering Azerbaijan branch, which is one of the leading engineering company under Tekfen Holding in Turkey.

Oluseun Oyeleke is a Master degree holder in Electronics and communication Engineering and a current $\mathrm{PhD}$ Student in Telecommunication. His focus is on wireless communication, Massive MIMO, MIMO and Machine learning for wireless telecommunication. He is currently an academic staff with the Air force Institute of Technology.

Ajoge Ohiani Joseph is a BSc holder in electrical and electronics engineering from eastern Mediterranean university north Cyprus(2014-2018). Interested in clean Energy Engineering.

Sadiq Thomas was born in 1980, he is the HOD of Computer Engineering Department Nile University of Nigeria, he also is a Senior Lecturer and the Research Group Team leader for the C\&E Engineering Research group at Nile University of Nigeria. He has several years experience in academics from time spent completing his BEng in Computer Engineering 2005 in the UK and Numerical Modelling of EMATs for his $\mathrm{PhD}$ Thesis also in the UK 2009. He has post-doctoral research and lecturing experience and has over 30 publications in International and National Journal and Conference proceeding. He is also a member of several National and International Engineering Bodies; COREN, IEEE, MIET and BiNDT 
How to cite this paper: Adegoke A. O, Oluseun D Oyeleke , Mahmud B, Ajoje J. O, Sadiq Thomase," Design and Construction of an Obstacle-Detecting Glasses for the Visually Impaired", International Journal of Engineering and Manufacturing(IJEM), Vol.9, No.4, pp.57-66, 2019.DOI: 10.5815/ijem.2019.04.05 